\title{
Prevalence of Smartphone Addiction and its Effects on Sub- Health and Insomnia: A Cross-Sectional Study Among Medical Students
}

\author{
Huan Liu \\ Yijishan Hospital of Wannan Medical College \\ Ming Zhang ( $\sim$ wnyxyzhangming@foxmail.com ) \\ Wannan Medical College \\ Zhiqing Zhou \\ Wannan Medical College \\ Long Huang \\ Wannan Medical College

\section{Ergang Zhu} \\ Wannan Medical College \\ Liang Yu \\ Wannan Medical College
}

\section{Research Article}

Keywords: prevalence, smartphone addiction, sub-health, insomnia

Posted Date: January 7th, 2022

DOI: https://doi.org/10.21203/rs.3.rs-1062413/v1

License: (c) (i) This work is licensed under a Creative Commons Attribution 4.0 International License. Read Full License 


\section{Abstract \\ Objective}

This study aimed to assess the Chinese medical students' smartphone addiction and its effects on sub-health and insomnia.

\section{Methods}

A cross-sectional survey was conducted from 14 October 2020 to 14 November 2020, by administering an online questionnaire to the students of Wannan Medical College.

\section{Results}

Of 2741 students who completed the survey, 1,447 (52.8\%) had smartphone addiction. Don't like the major( $\mathrm{p}=0.004)$, alcohol consumption $(p=0.001)$,bring smartphone to bed $(p=0.000)$, depression $(p=0.000)$ and anxiety $(p=0.000)$ strong associations of smartphone addiction. The impacts of smartphone addiction on sub-health $(\mathrm{p}=0.000)$ and insomnia $(\mathrm{p}=0.000)$ are significantly.

\section{Conclusion}

This survey shows that the smartphone addiction detection rate of medical students was $52.8 \%$. Don't like the major, alcohol consumption,bring smartphone to bed,depression and anxiety students had a higher smartphone addiction detection rate. The sub-health and insomnia of medical students are associated with smartphone addiction.

\section{Introduction}

In line with the developing economy,the number of people using smart phones has been growing tremendously in recent years and the smart phones have become an organic part of the everyday.Smartphones are powerful devices include many features such as phone, internet browserand social networks, playing games, emailing,communicating.According to a recent report, the current number of smartphone users is 3.8 billion in 2021, which accounts for approximately $48.20 \%$ of the global population owns a smartphone ${ }^{[1]}$.Moreover, today's college students are grow up with the company of smartphones, which have become a necessity in their lives ${ }^{[2]}$.Due to the convenience of smartphones, the abuse of smartphones has increased significantly during the COVID-19 pandemic.As the number of smart phones users increases, smartphone addiction has been rising as well.Several studies have reported that smartphone overuse can cause physical health problems for individuals such as musculoskeletal pain, blurred vision, headache and pain in the wrists or neck ${ }^{[3]}$. Moreover, excessive use of smartphones is associated with numerous negative outcomes, including poor academic performance, academic procrastination ${ }^{[4]}$, depression and anxiety,and poor sleep quality ${ }^{[5]}$. Research found that depressive and anxiety symptoms were highly prevalent among university students after the COVID-19 outbreak ${ }^{[6]}$.

Although smartphone addiction is a common public health problem and a high prevalence reported in previous studies, there is a severe lack of epidemiological data about smartphone addiction among medical students during the COVID-19 pandemic in China. It is known that the smart phones use in China is very common in medical students ${ }^{[7]}$. The medical profession is one of the most stressful areas of university education because of its strong professionalism and high academic requirements. There is an influence of smartphone addiction on sleep patterns,insomnia is important for health. The sub-health among medical students are also an issue of academic interest nowadays.Previous studies have already reported the relationship between smartphone addiction and sleep quality ${ }^{[8]}$.Overall, the evidence of sub-health, insomnia and smartphone addiction among Chinese medical students is completely lacking. 
The sub-health and sleep quality is severely affected in medical students due to high academic and clinical pressure.Use smartphones wisely plays a vital role in personal health. Although many studies on smartphone addiction have been conducted in China,to date, literature search showed there are no recent studies has investigated the relationship between smartphone addiction, sub-health and insomnia.Therefore, the purpose of this study were to determine the prevalence of smartphone addiction, and to clarify the association between smartphone addiction and sub-health among medical students in a College of Medicine in China.

\section{Materials And Methods}

\subsection{Participants and procedure}

This cross-sectional study web-based survey was conducted on the medical students of the Wannan Medical College between 14 October 2020 to 14 November 2020.The investigators of this survey were composed of undergraduates majoring in medicine from freshman to senior year of Wannan Medical College.We invited participants using the widely popular Chinese social networking "QQ" $₫ " W e C h a t "$ in the online survey through the Wenjuanxing platform (https://www.wjx.cn/app/survey.aspx).Counselors and student leaders survey data collection were recruited through the personal connections of the corresponding author of this article.Various professional counselors and student leaders assisted in completing this survey.According to the Declaration of Helsinki,the participants were informed of the purpose, signi ficance of this study . In this study, the participants scan the questionnaire QR code and filled out the questionnaire anonymouslyafter class. It took approximately 10 to 15 minutes for participants to complete the questionnaire.

\subsection{Measures}

\subsubsection{General demographic characteristics}

Participants' sociodemographic data, including sex, age, School year,Place of residence,daily smartphone use time,etc.

\subsubsection{Smartphone Addiction Scale (SAS).}

Smartphone AddictionTest is a validated instrument to measure smartphone addiction. It contains 10 -items which range from 1 (strongly disagree) to 6 (strongly agree), and cut-off scores are derived from 31/60 in males and 33/60 in females to shows the presence of smartphone addiction ${ }^{[9]}$. Cronbach's alpha coefficient of the Smartphone AddictionTest in this study was 0.81 .

\subsubsection{SHS evaluation}

The condition of SHS was measured by using the self-reporting questionnaire SHSQ-25[10], which is a widely used 25 -item selfreport questionnaire that evaluates subjective sub-health status in the previous three months. The 25 items yield seven component scores: fatigue,cardiovascular health, digestive tract, immune system , mental health.For each item, there are five response categories ( 1 = never or almost never, $2=$ occasionally, $3=$ often, $4=$ very often, and $5=$ always). In the data analysis, never or almost never to 0 , occasionally to 1 , often to 2 , very often to 3 and always to 4 . The sum of scores for these seven components yields the SHSQ-25 total score, which range from 0 to 100 .SHSQ-25 summed scores $\geq 35$ were defined as "suboptimal health", as has been demonstrated in the Chinese population. The Cronbach alpha coefficient of this scale in this survey was 0.834 .

\subsubsection{Insomnia Scale}

Insomnia symptoms were measured using the Athens Insomnia Scale (AIS),which consists of eight items. Each item of the scale was a four-point Likert scale from from 0 (no problem ) to 3 (very severe problem). The sum of scores of AIS is between 0 and 24 , the cutoff score of insomnia is $\left.6^{[11}\right]$.AIS were widely used and show good reliability and validity in the Chinese college population.In this present survey, the Cronbach's alpha for AIS was 0.90 .

\subsection{Statistical analyses}

Page $3 / 11$ 
Relationship between the demographic factors and the smartphone addiction was analyzed using the chi-square test.Logistic regression was used to explore the influencing factors of smartphone addiction.All $P$-values were two tailed,considering a $P$ $<0.05$ as statistically significant associated with smartphone addiction.

\subsection{Ethics}

This study design and procedure was approved by the ethics committee of Wannan Medical College.All participants provided their electronic informed consent for inclusion before participating in the study. These participants can withdraw at any time without providing any reason.

\section{Results}

\subsection{Demographic characteristics}

In the 2,741 students, a total of 1,447 (52.8\%) had smartphone addiction. Medical students with smartphone addiction tend to be Dissatisfied with school life $(\chi 2=35.822, p=0.000)$,Don't like the major $(\chi 2=41.717, p=0.000)$,Smoking $(\chi 2=6.822$, $p=0.009)$,Alcohol consumption $(\chi 2=17.605, p=0.000)$, Bring your phone to bed $(\chi 2=56.995, p=0.000)$, Use smartphones for longer time every day $(\chi 2=108.719, p=0.000)$ andSelf-perceived smartphone addiction $(\chi 2=368.997, p=0.000)$. The demographic characteristics of the study participants are presented in Table 1.

Table 1.Sociodemographic Characteristics of the study sample $\mathbb{N}=27410$ 


\begin{tabular}{|c|c|c|c|c|c|c|}
\hline \multirow[t]{2}{*}{ Variable } & \multirow[t]{2}{*}{ Category } & \multirow[t]{2}{*}{ Overall n(\%) } & \multicolumn{2}{|c|}{ smartphone addiction $\mathrm{n}(\%)$} & \multirow[t]{2}{*}{$x^{2}$} & \multirow[t]{2}{*}{ P-value } \\
\hline & & & No & Yes & & \\
\hline \multirow[t]{2}{*}{ Age } & $\leq 20$ & $1647(60)$ & $788 \rrbracket 47.8 \rrbracket$ & $859 \llbracket 52.2 \rrbracket$ & 0.669 & 0.413 \\
\hline & $\geq 21$ & $1094(40)$ & $506 \varangle 46.3)$ & $588(53.7)$ & & \\
\hline \multirow[t]{4}{*}{ School year } & 1 st year & 487(17.8) & $260 \rrbracket 53.4 \rrbracket$ & $227 \rrbracket 46.6 \rrbracket$ & 9.676 & 0.022 \\
\hline & 2nd year & $786(28.7)$ & $368 \rrbracket 46.8 \rrbracket$ & $418 \rrbracket 53.2 \rrbracket$ & & \\
\hline & 3rd year & $646(23.6)$ & $297 \rrbracket 46 \rrbracket$ & $349 \llbracket 54 \rrbracket$ & & \\
\hline & 4th year & $822(30)$ & $369 \rrbracket 44.9 \rrbracket$ & $453 \rrbracket 55.1 \rrbracket$ & & \\
\hline \multirow[t]{3}{*}{ Place of residence } & Rural & $1759(64.2)$ & $816 \rrbracket 46.4 \rrbracket$ & $943 \rrbracket 53.6 \rrbracket$ & 1.812 & 0.404 \\
\hline & Town & $574(20.9)$ & $274 \rrbracket 47.7 \rrbracket$ & $300 \otimes 52.3 \rrbracket$ & & \\
\hline & City & $408(14.9)$ & $204 \rrbracket 50.0 \rrbracket$ & $204 \llbracket 50.1 \rrbracket$ & & \\
\hline \multirow[t]{2}{*}{ Want to get a scholarship } & Yes & 2516(91.8) & $1192 \rrbracket 47.4 \rrbracket$ & $1324 \rrbracket 52.6 \rrbracket$ & 0.346 & 0.556 \\
\hline & No & 225(8.2) & $102 \rrbracket 45.3 \rrbracket$ & 123囚54.7ه & & \\
\hline \multirow[t]{2}{*}{ Student leader } & Yes & $809(29.5)$ & $395 \llbracket 48.8 \rrbracket$ & $414 \llbracket 51.2 \rrbracket$ & 1.204 & 0.273 \\
\hline & No & 1932(70.5) & $899 \llbracket 46.5 \rrbracket$ & $1033 \otimes 53.5 \rrbracket$ & & \\
\hline \multirow[t]{2}{*}{ Only-children } & Yes & $916(33.4)$ & $462 \searrow 50.4 \rrbracket$ & $454 \llbracket 49.6 \rrbracket$ & 5.751 & 0.016 \\
\hline & No & 1825(66.6) & $832 \rrbracket 45.6 \rrbracket$ & $993 \rrbracket 54.4 \rrbracket$ & & \\
\hline \multirow[t]{2}{*}{ In love } & Yes & $682(24.9)$ & $325 \llbracket 47.7 \rrbracket$ & $357 \rrbracket 52.3 \rrbracket$ & 0.072 & 0.788 \\
\hline & No & 2059(75.1) & $969 \rrbracket 47.1 \rrbracket$ & $1090 \otimes 52.9 \rrbracket$ & & \\
\hline \multirow[t]{3}{*}{ School satisfaction } & No & $186 \bigotimes 6.8)$ & $65 \rrbracket 34.9 \rrbracket$ & $121(65.1)$ & 35.822 & 0.000 \\
\hline & General & $1263(46.1)$ & $546(43.2)$ & $717 \rrbracket 56.8 \rrbracket$ & & \\
\hline & Yes & 1292(47.1) & $683(52.9)$ & $609(47.1)$ & & \\
\hline \multirow[t]{3}{*}{ Like the major } & No & 139(5.1) & $47(33.8)$ & $92(66.2)$ & 41.717 & 0.000 \\
\hline & General & $1081 \otimes 39.4 \rrbracket$ & $448(41.4)$ & $633(58.6)$ & & \\
\hline & Yes & $1521 \otimes 55.5 \rrbracket$ & $799(52.5)$ & $722(47.5)$ & & \\
\hline \multirow[t]{2}{*}{ Smoking } & Yes & $99 \llbracket 3.6 \rrbracket$ & $34(34.3)$ & $65(65.7)$ & 6.822 & 0.009 \\
\hline & No & $2642 \rrbracket 96.4 \rrbracket$ & $1260(47.7)$ & 1382(52.3) & & \\
\hline \multirow[t]{2}{*}{ Alcohol consumption } & Yes & $232 \bowtie 8.5 \rrbracket$ & 79(34.1) & 153(65.9) & 17.605 & 0.000 \\
\hline & No & $2509 \otimes 91.5 \rrbracket$ & $1215(48.4)$ & $1294(51.6)$ & & \\
\hline \multirow[t]{2}{*}{ Bring your phone to bed } & Yes & 1910ه69.7凶 & $811(42.5)$ & 1099(57.5) & 56.995 & 0.000 \\
\hline & No & $831 \otimes 30.3 \rrbracket$ & $483(58.1)$ & $348(41.9)$ & & \\
\hline \multirow[t]{4}{*}{ hours of daily smartphone usage } & $\leq 1$ hour & $73 \rrbracket 2.6 \rrbracket$ & $35 \llbracket 47.9 \rrbracket$ & $38 \rrbracket 52.1 \rrbracket$ & 108.719 & 0.000 \\
\hline & 2hours & $153 \llbracket 5.6 \rrbracket$ & $101(66.0)$ & $52(34.0)$ & & \\
\hline & 3hours & $463 \rrbracket 16.9 \rrbracket$ & $264 \rrbracket 57.0 \rrbracket$ & 199ه43.0》 & & \\
\hline & 4hours & $555 \rrbracket 20.2 \rrbracket$ & 294『53.0》 & $261 \rrbracket 47.0 \rrbracket$ & & \\
\hline
\end{tabular}




\begin{tabular}{|c|c|c|c|c|c|c|}
\hline & 5hours & $568 \rrbracket 20.7 \rrbracket$ & 280ه49.3》 & $288 \rrbracket 50.7 \rrbracket$ & & \\
\hline & $\geq 6$ hours & $929 \llbracket 33.9 \rrbracket$ & $320(34.40$ & $609(65.6)$ & & \\
\hline \multirow[t]{3}{*}{ Self-perceived smartphone addiction } & Yes & $753 \llbracket 27.5 \rrbracket$ & $161(21.4)$ & $592(78.6)$ & 368.997 & 0.000 \\
\hline & No & 1433囚52.3囚 & $912(63.6)$ & $521(36.4)$ & & \\
\hline & Not sure & $555 \llbracket 20.2 \rrbracket$ & 221(39.8) & $334(60.2)$ & & \\
\hline
\end{tabular}

$* p<0.05 ; * * p<0.01$

\subsection{Logistic regression for analysis of the factors associated with smartphone addiction}

Multivariate analysis demonstrated that smartphone addiction was significantly associated with like the major, alcohol consumption, bring your phone to bed, depression and anxiety. The associated factors of being at risk of smartphone addiction were don't like the major, alcohol consumption, bring your phone to bed, depression and anxiety (Table 2).

Table 2. Binary Logistic regression analysis of factors influencing smartphone addiction $\llbracket n=27410$.

\begin{tabular}{lllllll} 
Variables & $\beta$ & S.E. & Wald & $P$ & OR & OR 95\% Cl \\
\hline Like the major & & & 11.064 & 0.004 & & \\
\hline No & reference & & & & & \\
\hline General & -0.13 & 0.202 & 0.416 & 0.519 & 0.878 & $0.591-1.304$ \\
\hline Yes & -0.389 & 0.199 & 3.813 & 0.051 & 0.678 & $0.459-1.001$ \\
\hline Alcohol consumption $\otimes 1 \otimes$ & 0.508 & 0.155 & 10.764 & 0.001 & 1.661 & $1.227-2.250$ \\
\hline Bring your phone to bed(1) & 0.593 & 0.09 & 43.793 & 0.000 & 1.81 & $1.518-2.157$ \\
\hline Depression(1) & 0.842 & 0.114 & 54.728 & 0.000 & 2.321 & $1.857-2.901$ \\
\hline Anxiety(1) & 0.591 & 0.114 & 26.977 & 0.000 & 1.805 & $1.444-2.255$ \\
\hline Constant & -0.826 & 0.211 & 15.325 & 0.000 & 0.438 &
\end{tabular}

\subsection{Relationship between smartphone addiction and sub-health and insomnia}

As shown in Table 3, smartphone addiction people's sub-health was higher than not smartphone addiction $\left(r^{2}=0.365\right.$, $p<0.001)$, smartphone addiction people's insomnia was higher than not smartphone addiction $\left(r^{2}=0.566, p<0.001\right)$

Table 3. Pearson's correlation among Sub-health, Insomnia, and smartphone addiction

\begin{tabular}{|lllll|}
\hline variables & \multicolumn{2}{l}{ Sub-health } & \multicolumn{2}{l|}{ Insomnia } \\
\cline { 2 - 5 } & $\mathbf{r}$ & $\boldsymbol{P}$ & $\mathbf{r}$ & $\boldsymbol{P}$ \\
\hline smartphone addiction & $0.365^{\star \star}$ & 0.000 & $0.566^{\star \star}$ & 0.000 \\
\hline
\end{tabular}

\section{Discussion}

\subsection{Key findings}

As far as we know, this was the first study on the relationship between smartphone addiction and sub-health, sleep quality, anxiety, and depression among medical students. This study identified the prevalence of smartphone addiction, insomnia, and 
sub-health among medical students. The prevalence of smartphone addiction among the medical students in this study was $52.8 \%$. This is slightly higher than another study which found that $29.8 \%$ of medical students in China had smartphone addiction ${ }^{[12]}$. Previous studies have shown that smartphone addiction widespread among medical college students, and smartphone addiction has become a public health problem in China.According to a meta-analysis, the average prevalence of smartphone addiction among Chinese college students was approximately $23 \%{ }^{[13]}$.A study of Lebanese university students found that $49 \%$ of students had smartphone addiction ${ }^{[14]}$.

Smartphone are a "double-edged" sword that convenient our lives. The lives of many people and students are today increasingly influenced by new new technologies and devices, including smartphones. Due to the COVID-19 pandemic, young people had associated adverse psychological and behavioral effects, mainly related to infection control measures, which caused them to spend more time at home and mainly use technological tools.According to data released by the United Nations Educational Scientific and Cultural Organization (UNESCO), the COVID-19 pandemic is affecting nearly to 363 million students from the kindergartens to universities around the world ${ }^{[15]}$. In China, more than 220 million students took online study courses at home instead of traditional school teaching models due to the impact of the COVID-19 pandemic ${ }^{[16]}$. Smartphones provided to students the opportunity to continue schooling.Compared with older people, college students are usually psychologically immature and have poorer self-regulatory ability.Therefore, they are more likely to overuse smartphone ${ }^{[17]}$.

\subsection{The impact of smartphone addiction on sub-health}

Sub-health is a low-quality status between health and disease in the aspects of the physical, psychological and emotional performance of the subjects ${ }^{[18]}$. World Health Organization (WHO) defines the sub-health without organic pathological changes but functional changes as "the third state", also known as "chronic fatigue syndrome" ${ }^{[19,20]}$. Smartphone addiction are well known to be closely associated with sub-health.Studies had shown that long-term online, high-frequency online and Internet addiction are the important risk factors for SHS ${ }^{[21]}$, which is consistent with our research.During the COVID-19 pandemic, the smartphones could be more useful for many students as the main tool for communication, learning, entertainment, and information seeking. Excessive use of smartphones may cause sub-health, such as fatigue, indigestion, and

psychopathological problems, such as depression, and anxiety.Average time of the children and adolescents using smartphone per day during the COVID-19 pandemic was higher than before ${ }^{[22]}$.In fact, during the COVID-19 pandemic, students had more sleep disorders, ocular alterations, and musculoskeletal diseases than before. Pain on neck, shoulders, wrists and fingers are the musculoskeletal diseases related to smartphone overuse, reported more frequently ${ }^{[23]}$.Smartphone addiction may also result in diseases such as dry eye disease, burning sensation, conjunctival injection, decreased vision and macular degeneration ${ }^{[24,25]}$.

\subsection{The impact of smartphone addiction on insomnia}

Our research indicated that there was a strong association between smartphone addiction and insomnia. $(r=0.566)(P=0.001)$ (Table3).Studies had shown that college students with smartphone addiction were more likely to suffer from poor sleep quality. In a longitudinal study, Chen et al. reported that Insomnia and nocturnal awakening difficulties were predictors of smartphone addiction ${ }^{[26]}$.In addition, studies had shown that smartphone addiction was correlated with daytime sleepiness, reduced duration of night sleep ${ }^{[27]}$, later bedtime ${ }^{[27,28]}$ and poor sleep quality.First, excessive smartphones use at bed time might postpone or interfere sleep processes. Second, overuse of smartphones usually resulted in increased psychological stress, which also had a negative impact on sleep and physical recovery. Third, the blue light emitted by the screen might affect the melatonin levels and thus affect sleep and wakefulness. Finally, the electromagnetic fields emitted from smartphones might also be one of the reasons for the poor sleep quality.Due to the COVID-19 pandemic, students' sleep time and wake-up time were delayed during school closures.More and more evidence has shown that smartphone addiction is closely related to anxiety, depression, stress, impulsivity, and poor sleep quality ${ }^{[29-30]}$.

\subsection{Differences in smartphone addiction}

Based on interpersonal theory, individuals with high levels of smartphone addiction often neglect real-world social interactions, leading to less personal companionship and lower social support, which leads to more anxiety and depression.In this stage, 
college students are willing to share their inner world with others, resulting in they can gain social support. As shown in the current study, a high level of smartphone addiction was a positive indicator of anxiety and depression.Anxiety symptoms had a positive relationship with smartphone addiction ${ }^{[31]}$.The main characteristics of COVID-19 are universal susceptibility and strong infectivity. The number of confirmed and suspected patients had increased rapidly in a short period of time, putting people under a high degree of physical and psychological stress. In addition, in order to prevent the spread of the virus transmission, people are at home with restriction to outdoor activities such as, having dinner, traveling, and going to school,which aroused public fear and anxiety.In addition, the shortage of prevention materials ${ }^{[32,33]}$ at the beginning of the pandemic had caused panic and anxiety to the psychology of people.

Similarly, poor lifestyle behaviors such as; drinking and taking smartphone to bed are the main influencing factors of smartphone addiction.Adam summed up that the evening circadian typology was a risk factor ${ }^{[34]}$.Studies have also shown that adolescents with smartphone addiction have poor health ${ }^{[35]}$ and poor academic performance ${ }^{[36]}$. Some experts had found that the lifestyle changes brought about by the COVID-19 pandemic had allowed people to develop enhanced behaviors, such as video gaming, movie watching, using social media, and internet surfing ${ }^{[37,38]}$. Hysing et al. ${ }^{[39]}$ reported that students who go to bed between 10:00 and 11:00 PM had the best grade point averages, while those who go to bed after 11:00 PM had poorer grade point averages.

\subsection{Limitations}

This study has several limitations.First, all behavioral \sleep and smartphone data in this study was derived from self-reported questionnaire results $\llbracket$ which may inevitably have bias.Second, because all participants in this study are enrolled in the same school, our conclusions must be conservative. Therefore, we will conduct follow-up research in the future.

\section{Conclusion}

The smartphone addiction among Chinese medical students are a cause for concern because it may have a negative impact on their study, mood and physical health.Smartphone addiction has great impact on the sleep quality and sub-health among medical students. The results of this study revealed that like the major,alcohol consumption,Bring your phone to bed,depression and anxiety are related to smartphone addiction.

\section{Declarations}

\section{Ethics approval and consent to participate}

This study design and procedure was approved by the ethics committee of Wannan Medical College.All participants provided their electronic informed consent for inclusion before participating in the study. These participants can withdraw at any time without providing any reason. All data collection activities for this study were implemented with informed consent of participants. All methods were performed in accordance with the Declaration of Helsinki.

\section{Consent for publication}

All authors consent for publication.

\section{Availability of data and materials}

The datasets used and/or analyzed during the current study are available from the corresponding author $(\mathrm{H}$. L or M. Z) on reasonable request.

\section{Competing interests}

The authors declare that they have no competing interests or other interests that might be perceived to influence the results and/or discussion reported in this paper. 


\section{Funding}

This research was funded by MOE (Ministry of Education in China) Project of Humanities and Social Sciences ( 20YJC190006), The Teaching Quality and Teaching Reform Project of Anhui Provincial Department of Education (2020jyxm2076), School project of the University Student Mental Health Education Research Center of Wannan Medical College (SJD202110),Teaching reform project of Wannan Medical College(2020jyxm58),Teaching reform project of Wannan Medical College(2021zybz06) and the prevention and control science and technology emergency project for COVID-19 of Wuhu (2020rkx1-5).

\section{Author Contributions}

“Conceptualization, Huan Liu And Ming Zhang; methodology, Huan Liu; software, Zhiqing Zhou; validation, Long Huang, Huan Liu and Ming Zhang; formal analysis, Long Huang; investigation, Ergang Zhu; resources, Ming Zhang; data curation, Liang Yu; writing-original draft preparation, Huan Liu And Ming Zhang. All authors have read and agreed to the published version of the manuscript.

\section{Acknowledgements}

We would like to acknowledge all participants who taking time to respond to the survey. We express our gratitude to all the counsellors who assisted in the collection of this questionnaire.

\section{References}

1. BankMyCell. (2020). How many smartphones are in the world? Source: https://www.bankmycell.com/blog/how-manyphones-are-in-the-world. Accessed on August 5th, 2021.

2. Long J, Liu TQ, Liao YH, et al. Prevalence and correlates of problematic smartphone use in a large random sample of Chinese undergraduates. BMC Psychiatry. 2016 Nov 17;16(1):408.

3. Soni R, Upadhyay R, Jain M . Prevalence of smart phone addiction, sleep quality and associated behaviour problems in adolescents[J]. International Journal of Research in Medical Sciences, 2017, 5(2):515-519.

4. Mohamed SM, Mostafa MH. Impact of smartphone addiction on depression and self-esteem among nursing students. Nurs Open. 2020;7(5):1346-1353.

5. Alhassan AA, Alqadhib EM, Taha NW, et al. The relationship between addiction to smartphone usage and depression among adults: a cross sectional study. BMC Psychiatry. 2018;18(1):148.

6. Xie X, Zhu K, Xue Q, et al. Problematic Internet Use Was Associated With Psychological Problems Among University Students During COVID-19 Outbreak in China. Front Public Health. 2021;9:675380.

7. Zhang MWB, Lim RBC, Lee C, et al. Prevalence of internet addiction in medical students: a meta-analysis. Acad Psychiatry 2018;42:88-93.

8. Lemola S, Perkinson-Gloor N, Brand S,et al. Adolescents' electronic media use at night, sleep disturbance, and depressive symptoms in the smartphone age. J Youth Adolesc. 2015;44(2): 405-418.

9. Kwon M, Kim DJ, Cho H, et al. The smartphone addiction scale: Development and validation of a short version for adolescents. PLoS One. 2013;8:e83558.

10. Hou H, Feng X, Li Y, et al. Suboptimal health status and psychological symptoms among Chinese college students: a perspective of predictive, preventive and personalised health. EPMA J. 2018;9(4):367-377.

11. Soldatos C.R., Dikeos D.G., Paparrigopoulos T.J. The diagnostic validity of the Athens Insomnia Scale. J. Psychosom. Res. 2003;55:263-267.

12. Chen B, Liu F, Ding S, et al. Gender differences in factors associated with smartphone addiction: a cross-sectional study among medical college students. BMC Psychiatry. 2017;17(1):341. 
13. Tao, J., Luo, C., Huang, J.,et al.Meta-analysis of the current situation of mobile phone dependence among college students in China (in Chinese). Chinese Journal of School Health.2018,39(09):1391-1394.

14. Matar Boumosleh J, Jaalouk D. Depression, anxiety, and smartphone addiction in university students- A cross sectional study. PLoS One. 2017;12(8):e0182239.

15. United Nations Educational Scientific and Cultural Organization. With One in Five Learners Kept Out of School, UNESCO Mobilizes Education Ministers to Face the COVID-19 Crisis. (2020). Available online at: https://en.unesco.org/news/onefive-learners-kept-out-school-unesco-mobilizes-education-ministers-face-covid-19-crisis (accessed January 07, 2021).

16. Wang G, Zhang Y, Zhao J,et al. Mitigate the effects of home confinement on children during the COVID-19 outbreak. Lancet. (2020) 395:945-7.

17. Li L, Xu DD, Chai JX, et al. Prevalence of Internet addiction disorder in Chinese university students: A comprehensive metaanalysis of observational studies. J Behav Addict. 2018;7(3):610-623.

18. Li G, Xie F, Yan S,et al. Subhealth: Definition, criteria for diagnosis and potential prevalence in the central region of China. BMC Public Health 2013;13:446.

19. Cui WY. In: Investigation on the level of health quotient and subhealth status of undergraduate nursing students in school [master's thesis]. Changchun: Changchun University of Chinese Medicine; 2019. pp. 1-59.

20. Lee JC, Dong SO, Lee Y,et al. Recognition of and interventions for Mibyeong (subhealth) in South Korea: A national webbased survey of Korean medicine practitioners. Integrative Medicine Research 2014;3(2):60-66.

21. Ren L, Li J. Analysis of sub-health status and related factors of a high school student in Weinan City. Chin Sci Technol Inform. 2013;5:78-79.

22. Serra G, Lo Scalzo L, Giuffrè M, et al. Smartphone use and addiction during the coronavirus disease 2019 (COVID-19) pandemic: cohort study on 184 Italian children and adolescents. Ital J Pediatr. 2021;47(1):150.

23. Kim HJ, Kim JS. The relationship between smartphone use and subjective musculoskeletal symptoms and university students. J Phys Ther Sci. 2015;27(3):575-579.

24. Bergqvist UO, Knave BG. Eye discomfort and work with visual display terminals. Scand J Work Environ Health. 1994;20(1):27-33.

25. Lee HS, Park SW, Heo H. Acute acquired comitant esotropia related to excessive smartphone use. BMC Ophthalmol. 2016;16(1):37.

26. Chen YL, Gau SS. Sleep problems and internet addiction among children and adolescents: a longitudinal study. J Sleep Res. 2016;25:458-465.

27. Kawabe K., Horiuchi F., Oka Y., et al. Association between Sleep Habits and Problems and Internet Addiction in Adolescents. Psychiatry Investig. 2019;16:581-587.

28. Nakayama H., Ueno F., Mihara S.,et al. Relationship between problematic Internet use and age at initial weekly Internet use. J. Behav. Addict. 2020;9:129-139.

29. Demirci K, Akgönül M, Akpinar A. Relationship of smartphone use severity with sleep quality, depression, and anxiety in university students. J Behav Addict. 2015;4(2):85-92.

30. Thomée S. Mobile Phone Use and Mental Health. A Review of the Research That Takes a Psychological Perspective on Exposure. Int J Environ Res Public Health. 2018;15(12):2692.

31. Elhai JD, Levine JC, Hall BJ. The relationship between anxiety symptom severity and problematic smartphone use: a review of the literature and conceptual frameworks. J Anxiety Disord. (2019) 62:45-52.

32. Guan WJ, Ni ZY, Hu Y,et al. Clinical characteristics of coronavirus disease 2019 in China. New England Journal of Medicine 2020;382(18):1708-1720.

33. Pan A, Liu L, Wang C, et al. Association of public health interventions with the epidemiology of the COVID-19 outbreak in Wuhan, China. JAMA 2020;323(19):1915-1923.

34. Adan,Ana. A chronobiological approach to addiction. Journal of Substance Use, 2013, 18(3):171-183. 
35. Aarnio M, Winter T, Kujala U, et al. Associations of health related behaviour, social relationships, and health status with persistent physical activity and inactivity: a study of Finnish adolescent twins. Br J Sports Med. 2002 Oct;36(5):360-364.

36. Sharif I, Sargent JD. Association between television, movie, and video game exposure and school performance. Pediatrics. 2006;118(4):E1061-E1070.

37. Király O., Potenza M.N., Stein D.J., et al. Preventing problematic internet use during the COVID-19 pandemic: Consensus guidance. Compr. Psychiat. 2020;100:152180.

38. King D.L., Delfabbro P.H., Billieux J., et al. Problematic online gaming and the COVID-19 pandemic. J. Behav. Addict. 2020;9:184-186.

39. Hysing M, Harvey AG, Linton SJ, et al. Sleep and academic performance in later adolescence: results from a large population-based study. J Sleep Res. 2016 Jun;25(3):318-24.

\section{Supplementary Files}

This is a list of supplementary files associated with this preprint. Click to download.

- PrevalenceofsmartphoneaddictionanditseffectsonsubhealthandinsomniaAcrosssectionalstudyamongmedicalstudents.xlsx 